\title{
A Study on Throughput of LTE Multicast Systems under Co-existence Interference
}

\author{
S. Malisuwan, J. Sivaraks, N. Madan, and N. Suriyakrai
}

\begin{abstract}
LTE multicast has been recognized as an efficient technique to transmit a large volume of data to multiple mobile stations simultaneously. However, with the growth of wireless communications, it is common to find two different systems being deployed in the same area. This creates adjacent channel interference (ACI) which can result in significant reduction in neighbor system capacity. In this paper we adopt MATLAB simulations to investigate throughput degradation resulting from ACI. This paper also illustrates average throughput with different sizes of guard bands. Furthermore, simulations results conclude that without guard band, the average throughput level is unacceptable to operate LTE multicast system.
\end{abstract}

Index Terms-Throughput, LTE, multicast, co-existence, ACI.

\section{INTRODUCTION}

There are three types of multimedia communications between computers in a network namely, Unicast, Broadcast and Multicast. In most conventional Ethernet network, most Internet Protocol (IP) packets are sent via unicast (host to host) transmission. In a unicast communication, a computer packet is sent from one host to another single host. Unicast uses up bandwidth fast when sending large multimedia files. In the case of broadcasting, one computer communicates to multiple computers, where multimedia content is broadcasted to a large audience using a special broadcast address. In the traditional form of communication, broadcasting to a large audience is used, but in the modern day technological advances has altered user behavior. Today not every user is interested in receiving multimedia from a mass broadcast communication hence, resulting in waste of bandwidth. The third type of multimedia communication is multicast, which is popular in the current age because it corresponds with today's user behavior. With the explosive growth variety of multimedia services, the 3rd Generation Partnership Project (3GPP) has introduced the multimedia multicast service feature. Multicasting is ability to provide high definition multimedia content over IP-based networks from base station (BS) to a selected group of home users who are interested in receiving a particular content. These users will receive data packets from the same traffic at the same speed. Multicasting also optimizes the performance of the network as only one multicast data stream is sent out; therefore it preserves bandwidth on the network and eliminates any traffic redundancy. The users on multicast network are able to decide whether to listen to the multicast address so packets

Manuscript received March 1, 2015; revised June 15, 2015.

The authors are with the National Broadcasting and Telecommunications Commission Bangkok, Thailand (e-mail: \{settapong.m, jesada.s, navneet.m, nattakit.s\}@nbtc.go.th). are only sent to where they are required, hence, reducing load on network devices and enhanced efficiency is achieved from ability to control traffic on the network.

Rich multimedia services, such as mobile TV and mobile streaming, are considered of key importance for the LTE proliferation in the mobile market. The exponential data growth from smart phones has lead to the multimedia packet core network being pertinent in providing superior user experience and allowing rich data applications and services to be offered to users. Multimedia Multicast Services are envisaged to play an instrumental role to this end. In the modern day, there is a high demand for multicast as many emerging applications such as mobile TV and group oriented mobile commerce aim to deliver the same large volume of data to multiple users in the network [1].

As LTE is a technology that is based on next generation and is an all IP core network, it is suitable to conduct multicast on LTE network platform. A flexible technology, OFDMA-based LTE air interface supports both time division duplex (TDD) and frequency division duplex (FDD) modes in its PHY layer. For TDD mode, the system transmits the data frame-by-frame, and each 5-ms frame consists of a DL sub-frame and an UL sub-frame to prevent collisions between DL and UL transmissions [2].

FDD is duplexing scheme for the majority of cellular communication systems including IS-95, cdma2000, wideband code division multiple access (WCDMA), high speed downlink packet access (HSDPA), high speed uplink packet access (HSUPA), and LTE-FDD. Contrarily, the only popular TDD-based system that is widely used is TD-SCDMA [3].

TDD has several advantages over FDD which are flexible Downlink to Uplink ratio (i.e., TDD ratio), simpler and easier implementation of antenna techniques, and cheaper transceiver implementation. Therefore, TDD is also a duplexing scheme that is popular for wireless broadband systems such as IEEE 802.16e, IEEE 802.16m, and TD-LTE [3].

The exponential growth in wireless communications requires a flexible technology such as LTE that permits the deployment of two different systems in adjacent frequency bands in the same location. With technology neutrality being a worldwide standard, operators are being innovative by putting different mobile communication technologies into use hence, the current norm is such that multiple and different systems are more frequently located at the same site. This is called co-site, shared or co-existence which has a drawback that leads to increase in interference in such networks.

Interference on such networks further results in capacity degradation of both systems due to lack of RF isolation between two different communication systems [4].

For Adjacent Channel Interference (ACI) is when two 
systems operate in neighboring spectrum and interference cannot be entirely eliminated. The two main sources of ACI are out-of-band emission and spurious emission [5]. In calculations for the inter-system interference, the RF characteristics of transmitter and receiver are taken into account by weighting adjacent system signal with a parameter ACIR (Adjacent Channel Interference Power Ratio), which is defined as "the ratio of the total power transmitted from a source to the total interference power affecting a victim receiver, resulting from both transmitter and receiver imperfections"[6]. Therefore, as multicast is gaining tremendous popularity as a wireless multimedia communication technology, it is critical to study the coexistence interference for multicast wireless multimedia applications. Therefore, this study will focus on multicast experimenting ACI on different guard band including $0 \mathrm{MHz}$, $4 \mathrm{MHz}$ and $10 \mathrm{MHz}$.

\section{Average ThroughPUT OF MulticAst LTE Systems}

Multicast in access networks is using a base station (BS) or access point (AP) to broadcast data to a group of users, referred to as a multicast group. To ensure the signal reception quality at each user, one method is to adapt the data transmission rate to the worst channel among all users in the multicast group. It is inevitable that the data transmission rate will decrease when number of users' increases in the selected multicast group. On the other hand, in a system with fixed number of channels (e.g., subcarrier in OFDM systems) and fixed user population, the bandwidth resource allocated to a group is proportionate to the number of users in the group.

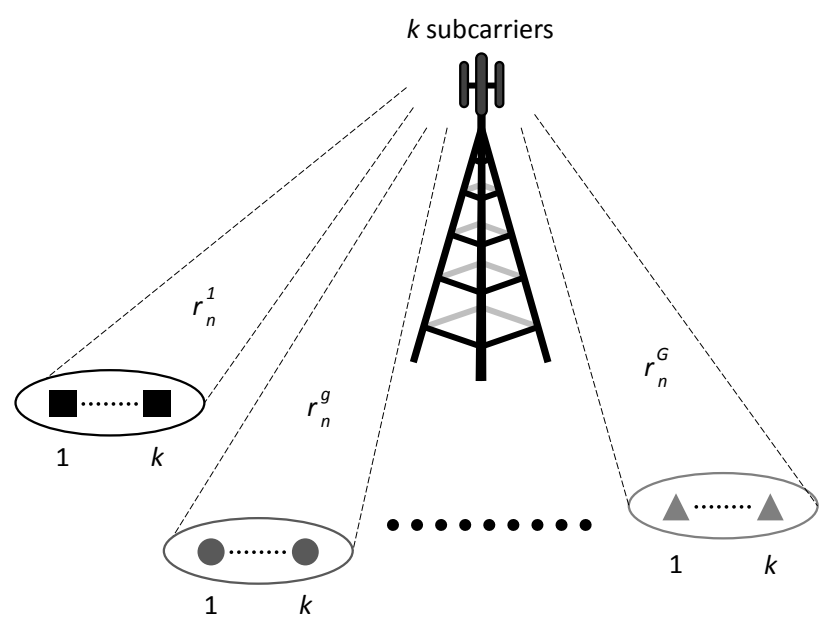

Fig. 1. LTE Multicast system: a base station (BS) broadcasts data to multicast groups.

Fig. 1 shows every multicast group with $k$ users is allocated $k$ fixed subcarriers in a wireless OFDM system. The BS transmits data to the users on subcarrier $n$ at a transmission rate $r_{n}^{g}$ for the $g$-th multicast group.

Consider a wireless OFDM multicast system with $J$ subcarriers and $K$ users requiring the same desirable program from the BS. The users are equally divided into $G$ multicast groups. Assuming that $K$ is divisible by $G$ and $J$ is an integer multiple of $K$, each multicast group is associated with $k=K / G$ users and $j k$ subcarriers, where $j=J / K$. For simplification, we assume $J=K$ in the rest of the paper, as shown in Fig. 1. Our results, however, easily extends to the case with $j>1$. We further assume that equal power is transmitted on all subcarriers.

Let $S C_{m, n}^{g}(g=1, \cdots, G ; m=1, \cdots, k ; n=1, \cdots, k)$ denote the sub-channel of user $m$ of the $g$-th multicast group on subcarrier $\mathrm{n}$ with a stationary and ergodic time-varying power gain $\alpha_{m, n}^{g}$. The power gains $\alpha_{m, n}^{g}$ for all the $m$ and $n$ are assumed to follow an i.i.d. standard exponential distribution (Rayleigh fading). It is assumed that a perfect instantaneous channel estimation is made in every user's receiver and is transmitted back to the BS through error free feedback path. So the channel gain $\alpha_{m, n}^{g}$ is known both to the BS and the receiver. Let $P$ denote the average transmit signal power on each subcarrier, $N_{0}$ denote the singlesided power spectral density of Additive White Gaussian Noise (AWGN), and $B$ denote the bandwidth of each subchannel. Let $\gamma_{t}=P /\left(N_{0} B\right)$ denote the average transmitterside SNR. Then the instantaneous received SNR of $S C_{m, n}^{g}$ is represented by $\gamma_{m, n}^{g}=\alpha_{m, n}^{g} P\left(N_{0} B\right)=\alpha_{m, n}^{g} \gamma_{t}$.

Based on Shannon's channel capacity, the instantaneous capacity of each sub-channel $S C_{m, n}^{g}$ can be represented by:

$$
B \log _{2}\left(1+\gamma_{m, n}^{g}\right)=B \log _{2}\left(1+\alpha_{m, n}^{g} \gamma_{t}\right)
$$

In this paper, we adopt the average throughput of one user which is derived in [7]. By assuming each user perceives i.i.d. Rayleigh fading channels, the average throughput of one user is given by:

$$
C=k \int_{\alpha} B \log _{2}\left(1+\gamma_{t} \alpha\right) p(\alpha) d \alpha
$$

where substituting all the i.i.d. $\alpha_{n}^{g}$ with the variable $\alpha$ that has the probability distribution $p(\alpha)$.

Given $\gamma_{t}$ and $B$, the user's average throughput $C$ is re-written as a function of the size of a multicast group, $k$,

$$
C(k)=\frac{B k}{\ln 2} e^{k / \gamma_{t}}\left(\int_{k / \gamma_{t}}^{\infty} e^{-z} \text { in } z d z-e^{-k / \gamma_{t}} \ln \frac{k}{\gamma_{t}}\right)
$$

As $C(k)$ increases with $k$, each user can enjoy a higher throughput when users are divided into larger multicast groups. In this regard, the best grouping strategy is to put all users requiring the same program into one multicast group [7].

\section{CO-EXISTENCE INTERFERENCE IN LTE SYSTEMS}

Adjacent channel interference (ACI) is interference between links that communicate geographically close to each 
other using neighboring frequency bands. For instance, several network operators may deploy their own networks in the same area and operate on frequency bands that are close to each other. Hence, ACI needs to be taken into account in the system specifications so it does not hamper the system performance. In practice, the minimum coupling loss between a transmitter and a receiver on adjacent bands may be estimated and the transceivers can designed such that ACI remains at a tolerable level. In order to do so the transceivers must utilize adequate filtering on stop-bands. The needed quality of the filters may be controlled by reserving a guard band between the bands.

A related interference type to ACI is coexistence interference which occurs among heterogeneous radio access technologies. Coexistence Interference may arise on the same frequency band or between adjacent frequency bands.

FDD and TDD indicate the duplex division method type for uplink and downlink. FDD is the duplex method to assign separate frequency bands for uplink transmission and for downlink transmission, thus FDD requires the paired band. A frequency separation between uplink and downlink transmissions is required due to the characteristic of FDD and known as 'Duplex spacing'. Generally, FDD downlink band is assigned as the upper band of the pair. TDD is the duplex method to assign alternate time slots for uplink and downlink transmission on the same frequency in an assigned band [8].

In the $2.6 \mathrm{GHz}$ band, the $3 \mathrm{GPP}$ band 7 is defined by assigning the lower $70 \mathrm{MHz}$ of the spectrum, from $2.50 \mathrm{GHz}$ to $2.57 \mathrm{GHz}$, to UpLink and the upper $70 \mathrm{MHz}$, from $2.62 \mathrm{GHz}$ to $2.69 \mathrm{GHz}$, to DownLink.

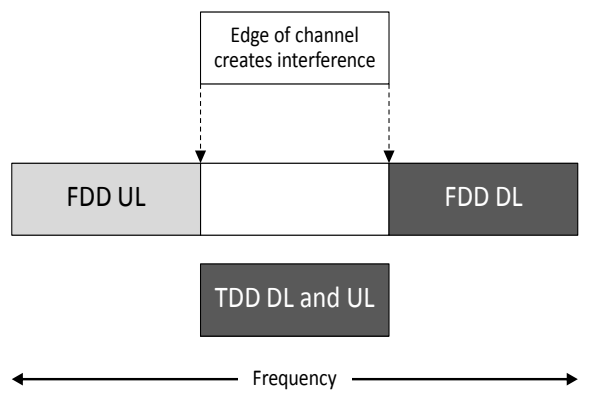

Fig. 2. Band allocation of TDD and FDD.

Fig. 2 shows that both edge channels of the TDD band at $2.57 \mathrm{GHz}$ and $2.62 \mathrm{GHz}$ can create interference from the adjacent edge channel of the FDD UL and DL, which can impact the co-existence of LTE FDD and TDD [9].

To minimize the possible interference, a specific spectrum gap may be used to provide isolation. Isolation can also be achieved with a combination of provisions including guard band allocation, antenna separation, and filtering. In order to assess the co-existence interference incurred between LTE cells, the model in [10] is used.

In this paper, the methodology to calculate the adjacent channel interference and co-existence interference adopts from [10]. We consider circular cells as shown in Fig. 3 and assume that the links from the interfering BSs are mutually independent. $B S_{0}$ is the serving $\mathrm{BS}$ and $M S_{0}$ is the victim MS. The relationship between the parameters are depicted in Fig. 3 transmitted power of serving cell $B S_{0}\left(P_{t_{0}}\right)$ and neighbour cell $B S_{1}\left(P_{t_{1}}\right)$, received power at victim $M S_{0}\left(P_{r}\right)$, interference power $\left(I_{a d}\right)$, the distance between $B S_{0}$ and $B S_{1}$ $\left(d_{0}\right), B S_{0}$ and $M S_{0}\left(d_{1}\right)$, and $B S_{1}$ and $M S_{0}\left(d_{2}\right)$, respectively.

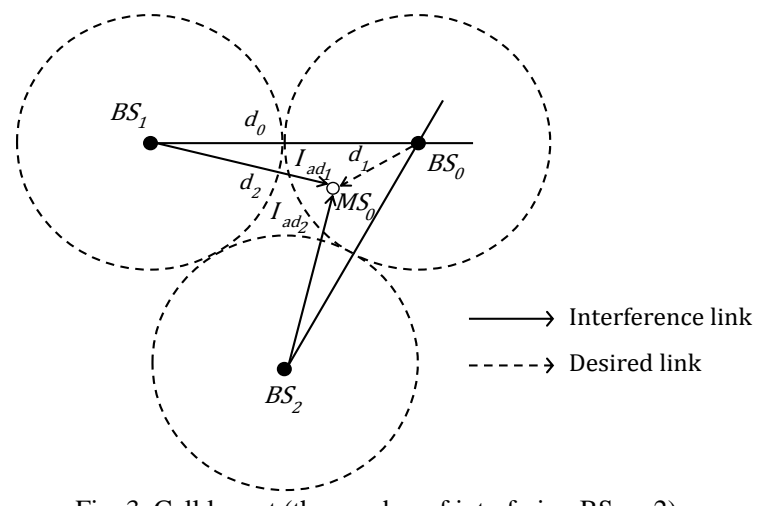

Fig. 3. Cell layout (the number of interfering BSs = 2).

Let $\xi_{D}$ and $\xi_{I}$ be the random component due to shadowing on the desired link and the interference link, respectively, $\gamma$ be the path-loss exponent and $\kappa_{I}$ be the adjacent channel interference ratio (ACIR) in decibels. Then, the interference power $I_{a d}$ is modeled [10] by :

$$
I_{a d}=10^{\frac{P_{r}}{10}}\left(\frac{d_{1}}{d_{2}}\right)^{\gamma} 10^{\frac{\xi_{D}-\xi_{I}-\kappa_{I}}{10}}
$$

The interference scenario in Fig. 3 studied in [10]. The research results in [10] presents level of ACI and shows the relationship between guard-band sizes and statistical values that we can use for the simulations in the next section.

A parameter named ACIR (adjacent channel interference ratio) is used to measure the overall ACI and defined as the ratio of the total power transmitted from an aggressor transmitter to the total interference power affecting a victim receiver in the adjacent channel. The ACIR is expressed as a combination of ACLR and ACS according to the following equation [11].

$$
A C I R=\frac{1}{\frac{1}{A C L R}+\frac{1}{A C S}}
$$

In this paper, we assume that ACIR is equal to ACLR by assuming $\mathrm{ACS}=\infty$. We use information of a spectrum emission mask of MS and BS for LTE system from [12], [13]. With the information of this mask, ACLR is calculated by $P_{T X_{\text {inband }}} / P_{T X_{\text {outband }}}$, where $P_{T X_{\text {inband }}}$ is the transmission power of interfering BS and $P_{T X_{\text {outhand }}}$ is the received power of victim MS, respectively. Let $\alpha$ be a guard-band size, we can obtain $P_{T X_{\text {outband }}}$ as follows:

$$
P_{T X_{\text {outbond }}}= \begin{cases}\int_{\alpha}^{5} 10^{\frac{3-7 / 5 \alpha}{10}} d a+10^{-0.4} \times 5+10^{-1.3} \times \alpha, & 0<\alpha \leq 5, \\ 10^{-0.4} \times(1-\alpha)+10^{-1.3} \times \alpha, & 5<\alpha \leq 10 .\end{cases}
$$

In the next section, we take the ACI in to the account to 
evaluate the average throughput by MATLAB simulations.

\section{Simulation AND Results}

In this section, the average throughput of LTE multicast systems under co-existence interference (ACI) is evaluated. The comparison of the average throughput between three different guard band parameters $(0,4 \mathrm{MHz}$ and $10 \mathrm{MHz})$ when the number of interfering $B S_{s}=2(N=2)$ is carried out.

The essential parameters for the simulations in this paper are used from the reference [7], [10]. The cell radius of $R=100 \mathrm{~m}, d_{0}=5 \mathrm{~m}, \gamma=3, P_{r}=-111 \mathrm{dBm}$ and the group multicast size $k=100$ are used in the simulations.

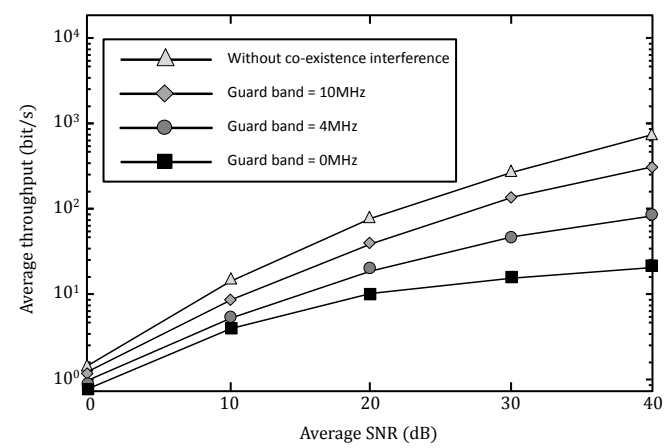

Fig. 4. Average throughput comparison.

In this simulation, the average throughput is plotted with different sizes of guard band; $0 \mathrm{MHz}, 4 \mathrm{MHz}$ and $10 \mathrm{MHz}$. It is shown that the average throughput increases with the guard band size. Fig. 4 describes that LTE multicast is an effective system with enough guard band size. It can be explained that when the guard band increases, the adjacent channel interference decreases. In this case, we can roughly conclude that $10 \mathrm{MHz}$ guard band is suitable to apply to protect the co-existence interference (ACI) for this LTE multicast system.

Without guard band (guard band $=0 \mathrm{MHz}$ ), the average throughput level is unacceptable to operate the LTE multicast system.

\section{CONCLUSION}

Multicast communication addresses packets so it is only sent to where it is required, hence reducing a load on network devices and results in enhanced efficiency in traffic control on the network. In this research we consider multicast in access networks and results provided is plotted with different sizes of guard band including $0 \mathrm{MHz}, 4 \mathrm{MHz}$ and $10 \mathrm{MHz}$. When the guard band increases, the adjacent channel interference decreases. It can be concluded that $10 \mathrm{MHz}$ guard band is suitable to protect co-existence ACI for LTE multicast system. However, with guard band equivalent to 0 $\mathrm{MHz}$, the average throughput is unacceptable and cannot operate on LTE multicast system.

\section{REFERENCES}

[1] U. Varshney, "Multicast over wireless networks," Communications of the ACM, vol. 45, pp. 31-37, December 2002.

[2] J. J. Jiang and G. Klein, "Risks to different aspects of system success," Information \& Management, vol. 36, pp. 263-272, November 1999.
[3] R. Zheng, X. Zhang, X. Li, Y. Fang, and D. Yang, "On the Wimax system co-existence with Wcdma and Cdma2000 1x system," in Proc. 4th International Conference on Wireless Communications, Networking and Mobile Computing, 2008, pp. 1-5.

[4] W. Jiang, L. Shuangchun, N. Kai, and W. Weiling, "Capacity loss due to coexistence of Wcdma and Cdma2000 systems," School of Information Engineering, Beijing University of Posts and Telecommunications, 2003.

[5] Universal Mobile Telecommunications System (Umts) Radio Frequency (Rf) System Scenarios. [Online]. Available: http://www.etsi.org/deliver/etsi_tr/125900_125999/125942/06.04.00_ 60/tr_125942v060400p.pdf

[6] R. Zheng, X. Zhang, X. Li, Y. Hai, and D. Yang, "Coexistence study in the 2500-2690 Mhz band between Wimax and Wcdma systems," in Proc. IEEE 68th Vehicular Technology Conference, 2008, pp. 1-6.

[7] J. Liu, W. Chen, Z. Cao, Y. Zhang, and S. C. Liew, "Asymptotic throughput in Wireless Multicast of DM Systems," in Proc. IEEE 2008 Global Telecommunications Conference, 2008, pp. 1-5.

[8] WIMAX Forum. (November 4). Managing Tdd-Fdd Interference Between Co-Sited Base Stations Deployed In Adjacent Frequency Blocks. [Online]. Available: http://resources.wimaxforum.org/sites/wimaxforum.org/files/documen t_library/FDD-TDD_Interference_analysis_involving_WiMAX_syste ms_Final_091103.pdf

[9] WIMAX Forum. (2011). Requirements for Wimax Coexistence with LTE Network. [Online]. Available: http://resources.wimaxforum.org/sites/wimaxforum.org/files/technical _document/2012/05/WMF-T31-132-v01-WiMAX_LTE_Coexistence. pdf

[10] Y. S. Choi, J. H. Wui, and D. Kim, "Statistical analysis of adjacent channel interference in LTE downlink," presented at International Symposium Antennas and Propagation (ISAP), Jeju, Korea, Oct. 25-28, 2011.

[11] W.-Y. Yeo, S.-H. Moon, and J.-H. Kim, "Uplink scheduling and adjacent-channel coupling loss analysis for Td-LTE deployment," The Scientific World Journal, vol. 2014, pp. 1-15, 2014.

[12] 3GPP, "3rd Generation Partnership Project; Technical Specification Group Services And System Aspects: Telecommunication Management; Evolved Universal Terrestrial Radio Access Network (E Utran) Network Resource Model (Nrm): Integration Reference Point (IRP): Information Service (Is) (Release 8)," 2010.

[13] 3GPP, "LTE; Evolved Universal Terrestrial Radio Access (E-Utra); Base Station (Bs) Radio Transmission And Reception (3GPP Ts 36.104 Version 9.4.0 Release 9)," 2010.

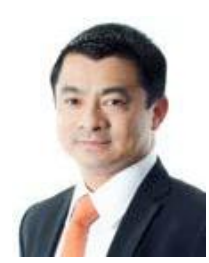

Settapong Malisuwan was born in 1966, in Bangkok, Thailand. He received his $\mathrm{PhD}$ in electrical engineering (telecommunications), specializing in EMI/EMC from Florida Atlantic University (State University System of Florida), Boca Raton in 2000. He received his MSc in electrical engineering in mobile communications system, from George Washington University in 1996 an MSc in electrical engineering from Georgia Institute of Technology in 1992 and a BSc in electrical engineering from the Chulachomklao Royal Military Academy, Nakhon-Nayok, Thailand in 1990 He served in the Royal Thai Armed Forces for more than 25 years. His research interests are in efficient spectrum management and Telecommunications policy and management. Dr. Settapong Malisuwan is currently the Elected Vice Chairman and Board Member in the National Broadcasting and Telecommunications Commission, Thailand.

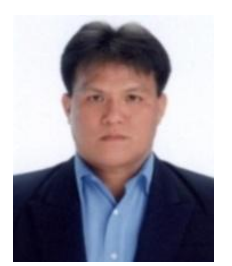

Jesada Sivaraks was born in 1970, in Bangkok, Thailand. He received his MSEE degree from Oklahoma State University in 1996 and BEng from King Mongkut's Institute of Technology, Thailand. He completed his $\mathrm{PhD}$ in electrical engineering at Florida Atlantic University, Boca Raton, FL in 2001. Since 2011, he has been working in national broadcasting and telecommunications commission as the secretary to the vice chairman. His PhD work is on the system aspects of Bluetooth, WLAN and mobile IP/CDPD. His current research interests are in telecommunication planning and related system analysis and efficient spectrum management. He is a member of Tau Beta Pi, Florida Epsilon and was an Honorary Advisory's Chairman of Science \& Technology committee of Parliament in 2009 


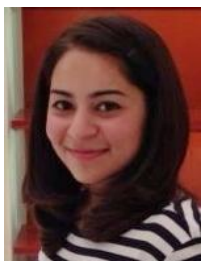

Navneet K. Madan was born in Bangkok, Thailand, in 1987. She received her bachelor of business administration in international business management from Mahidol University in 2008, and received her master of science degree in strategic management and marketing, Middlesex university, London, United Kingdom. She has been working as an assistant to vice chairman in National Broadcasting and
Telecommunications, Bangkok, Thailand since January 2012. Her research interests are in spectrum management strategic flexibility, market orientation and environmental uncertainty in fast clockspeed industries.

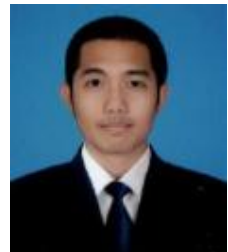

Nattakit Suriyakrai was born in Khonkhaen, Thailand, in 1987. He received his bachelor of liberal arts in Japanese Language from Thammasat University in 2010. He has been working as an assistant to vice chairman in National Broadcasting and Telecommunications, Bangkok, Thailand since November 2012. His research interests are in technology management and spectrum management. 